\title{
High precision model of precession and nutation of the asteroids (1) Ceres, (4) Vesta, (433) Eros, (2867) Steins, and (25143) Itokawa
}

\author{
A. Petit ${ }^{1}$, J. Souchay ${ }^{1}$, and C. Lhotka ${ }^{2}$ \\ 1 Observatoire de Paris, SYRTE, CNRS/UMR 8630, 75014 Paris, France \\ e-mail: alexis.petit@unamur.be; jean.souchay@obspm.fr \\ 2 Department of Mathematics, University of Rome Tor Vergata, 00133 Rome, Italy \\ e-mail: christoph.1hotka@univie.ac.at
}

Received 23 October 2013 / Accepted 26 February 2014

\begin{abstract}
Context. Several asteroids have recently been visited by spacecrafts that give us precious information and new constraints on their physical and rotational properties. In parallel, there are already several well-established theories to model the rotational motion of a rigid body, but accurate models of the rotational motion of asteroids have been poorly investigated so far.

Aims. We aim to model the rotational motion of the asteroids (1) Ceres, (4) Vesta, (433) Eros, (2867) Steins, and (25143) Itokawa with high precision. Their physical parameters have been or will be (in the case of Ceres) better constrained by data from space missions. We concentrate in particular on the motion of their spin axis in space, a component that is generally not studied.

Methods. We used Kinoshita's theory, based on a Hamiltonian approach for the rotation of a rigid body. We deduced an analytical model for determining the precession rate and the nutation coefficients for the set of five asteroids selected above.

Results. For each asteroid considered we make a summary of the rotational and physical characteristics necessary for our calculations, and we give both the precession rate and accurate tables of nutation. Results show a very high precession rate for (25143) Itokawa, of more than one degree per decade $\left(\dot{\psi}=-(461.52 \pm 6.57)^{\prime \prime} / \mathrm{yr}\right)$, and rather high ones for (433) Eros and (2867) Steins. We present the complete tables of nutation for both (1) Ceres and (4) Vesta, and give the peak-to-peak amplitudes for the five asteroids. At last, we show the curves described by the spin axis in space. Results agree with previous ones for (4)Vesta and (433) Eros.

Conclusions. In this paper we show that it is possible to accurately compute the combined precession-nutation motion of asteroids that are well constrained by observational data as we do for a set of five asteroids. This enables one to understand the rotational behavior of their spin axis in space and constitutes an interesting step toward knowing of their rotational evolution and its consequences on their history. This work should serve as a basis for more extended ones dealing with the long-term rotational dynamics of the asteroids studied.
\end{abstract}

Key words. celestial mechanics - minor planets, asteroids: general - planets and satellites: fundamental parameters

\section{Introduction}

With the drastic improvement in ground-based observations as well as through very accurate in situ observations from space probes, our knowledge of asteroids and minor planets has increased significantly in the past twenty years. Up to now nine asteroids or minor planets have already been visited by spacecrafts.

The first mission, Galileo, was launched on 1989 Oct. 18 with Jupiter as a target. It flew over (951) Gaspra and (243) Ida, at the same time discovering its moon Dactyl. The NEAR probe was launched on 1996 Feb. 17, then passed close to (253) Mathilde and finished its trajectory on (433) Eros. The orbital operation around (433) Eros lasted longer than one year and our knowledge of its characteristics are more accurate with respect to other space missions. The Deep Space probe was launched on 1998 Oct. 24, and encountered (9969) Braille before joining the comet Borrelly. After these various successful missions, the Hayabusa spacecraft was launched on 2003 May 9, for landing on the surface and collecting samples of the asteroid (25143) Itokawa. Notice that today, a second Hayabusa mission is in preparation by JAXA for a launch in 2014 toward the asteroid (162173) $1999 \mathrm{JU}_{3}$. The spacecraft Rosetta, whose main objective is to join the comet $67 \mathrm{P} /$ ChuryumovGerasimenko in 2014, has encoutered the asteroids (2867) Steins and (521) Lutetia. Recently, (4) Vesta has been visited by the DAWN spacecraft for one year, and (1) Ceres will be its next target for 2015.

Thanks to these various space missions, we could get precious determinations of a set of parameters related to the physical properties, the composition, and the internal structure of the celestial bodies that have been explored. In particular, shape, mass, rotation period, and moments of inertia determined with a relatively high precision during flyby or orbital trajectory are fundamental parameters for studing the rotational motion of the asteroids that are concerned. This aspect of the dynamics will give us a new line of thought about their formation and their evolution.

In this paper we determine analytically and for a short time scale the motion of the spin axis of five asteroids explored by the space probes that we have mentioned above, under the gravitational torque exerted by the Sun. These objects have well constrained physical and rotational parameters, which allow accurate analytical modeling. A few works have already treated this problem, for instance, those recently done in the case of (1) Ceres by Rambaux et al. (2011) from Eulerian approach or Lara et al. (2011) from a Hamiltonian one. For our study, we use the Hamiltonian approach in the following, which has already 
been applied by Souchay et al. (2003a,b) when they calculated the free and forced rotational motion of (433) Eros. In light of new data, we are able to give the precession rate of the spin axis and the leading nutation coefficients in longitude and in obliquity with relatively high accuracy, for the set of asteroids mentioned above with well-known physical and rotational parameters. Thus we will have a good representation on a short time scale of the rotational motion of these celestial bodies.

\section{The forced motion}

We consider here the forced rotational motion of the asteroids, more precisely the motion of their spin axis undergoing motion caused by gravitational external torque exerted by the Sun. In the following, we have to define the action-angle variables that characterize the rotation and the reference frames related to the problem. Then, we can express the equations of motions in a Hamiltonian formulation.

\subsection{Variables and reference frames}

At first approximation we consider each asteroid as a rigid body. For studing of the forced rotational motion of a rigid body, we can refer to the complete Kinoshita's theory already applied with success for the Earth at the milliarsecond level (Kinoshita 1977) and at the microarsecond level (Souchay et al. 1999), as well as for Mars (Bouquillon et al. 1999) and Venus (Cottereau et al. 2010).

Here, we focus on the motion of the axis of angular momentum with respect to an inertial reference frame. Indeed, we postulate that, at first approximation and as is the case for the Earth and the other terrestrial planets, the axis of rotation and the axis of figure of the asteroid are very close to the axis of the angular momentum, and that their motions of precession and nutation in space are quasi-identical. In the case of the Earth, the validity of these postulates can be checked easily (Kinoshita 1977; Souchay et al. 1999).

The reference frame is arbitrary by nature. Nevertheless, since we are interested in particular by the evolution of the obliquity, we choose the orbital plane of the asteroid as the reference plane at a given epoch (J2000.0). For a study at a relatively short time interval ( $\approx 100 \mathrm{yrs}$ ), the orbital elements do not change significantly (except for true anomaly), and we can consider the orbital plane as quasi fixed during this interval. Otherwise, we would have to consider a complementary Hamiltonian term $E$ related to the slow motion of the orbital plane in the expression of the Hamiltonian $K$ characterizing the rotation of the asteroid (Kinoshita 1977). Here, $K$ is expressed with the help of the Andoyer canonical variables $(l, g, h, L, G, H)$ that are described in detail by Kinoshita (1977).

Here we are particularly concerned with the angle-action couple of variables $h$ and $H$ where $h$ is the precession angle of the angular momentum axis and $H$ the projection of the angular momentum axis on the polar axis normal to the reference plane. From this variable $H$, we have access to the obliquity of the angular momentum axis with respect to the inertial plane, thanks to the basic relationship:

$H=G \sin I$

where $G$ is the amplitude of the angular momentum and $I$ is the obliquity. These parameters are shown in Fig. 1. We underline that in the Kinoshita notation, we have the convention $\psi=-h$ and $I=-\epsilon$.

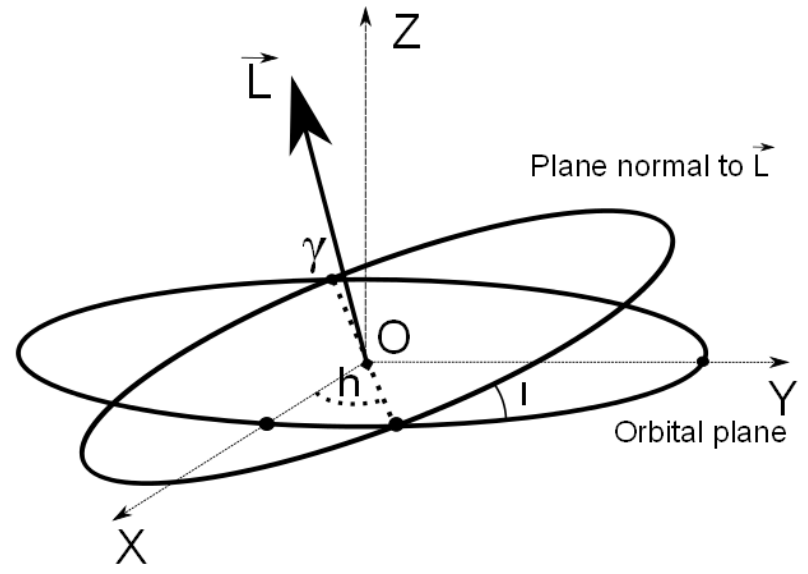

Fig. 1. Angular-momentum vector $\boldsymbol{L}$ with the help of the precession angle $h$ and of the obliquity $I$ with respect to inertial plane $(O, X, Y)$. By analogy with the Earth, the vernal point $\gamma$ is the ascending node of the asteroid orbital plane with respect to the mean equator.

\subsection{Equations of motion}

From Kinoshita (1977), we can express the equations of motion for the axis of the angular momentum in a straightforward way, through the following equations:

$$
\left\{\begin{array}{l}
\frac{\mathrm{d} h}{\mathrm{~d} t}=\frac{1}{G \sin I} \frac{\partial K}{\partial I} \\
\frac{\mathrm{d} I}{\mathrm{~d} t}=-\frac{1}{G \sin I} \frac{\partial K}{\partial h}
\end{array}\right.
$$

where the Hamiltonian can be written as $K=F_{0}+E+U$ with $F_{0}$ the torque-free motion of rotation, $E$ the component due to the motion of the orbital plane, and $U$ the disturbing function due to the action of the Sun. The free part is not a function of variables of motion $I$ and $h$, and it is not treated here. Here, $E$ can be neglected because the variation of the orbital elements is not significant on a short time scale as we have explained above.

We can give an analytical expression of the precessionnutation motion. Using Hori's averaging method (Hori 1966), we can decompose our perturbing potential $U_{\text {Sun }}$ due to the Sun into two parts: its secular one $U_{\text {Sun }}^{\text {sec }}$ and its periodic one $U_{\text {Sun }}^{\text {per }}$. Then we introduce $W_{1}$ as an average function that eliminates short-period terms at the first order. Here, $W_{1}$ is defined by Lie's method as: $W_{1}=\int U_{\text {Sun }}^{\text {per }} \mathrm{d} t$.

We express the precession rate $\dot{\psi}=-\dot{h}$ as the effect of $U_{\mathrm{Sun}}^{\mathrm{sec}}$, the secular part of the potential due to the Sun:

$\dot{\psi}=-\dot{h}=\frac{1}{G \sin I} \frac{\partial U_{\text {Sun }}^{\mathrm{sec}}}{\partial I}$

The nutation motion in longitude $\Delta \psi=-\Delta h$ and in obliquity $\Delta \epsilon=-\Delta I$ are given by the following two relationships:

$$
\left\{\begin{aligned}
\Delta \psi & =\frac{1}{G \sin I} \frac{\partial W_{1}}{\partial I} \\
\Delta \epsilon & =-\frac{1}{G \sin I} \frac{\partial W_{1}}{\partial h} .
\end{aligned}\right.
$$

Thanks to these equations and following the same procedure as Souchay (2003a) in the case of (433) Eros for the expression of the potential of the Sun, we finally express the rotational changes through $\dot{\psi}, \Delta \psi$, and $\Delta \epsilon$ analytically. 


\section{Expression of rotational motion}

We assume that each asteroid that is studied in this paper can be approximated by a perfect rigid ellipsoid body with semi-axes a, b, c satisfying $a>b>c$ and three moments of inertia $A, B, C$ satisfying $A<B<C$. The way of determining of these moments of inertia will be developed in the Sect. 4.4. Here, we present the analytical formulation of the rotational motion that can be expressed as a sum that cosine and sine of the mean anomaly.

\subsection{Expression of the potential}

The disturbing potential due to the Sun can be expressed by expansions in spherical harmonics (Kinoshita 1977):

$$
U_{\text {Sun }}=\frac{\kappa^{2} M_{\mathrm{s}}}{r^{3}}\left[\frac{2 C-A-B}{2} P_{2}^{0}(\sin \delta)+\frac{A-B}{4} P_{2}^{2}(\sin \delta) \cos [2 \alpha]\right]
$$

where $\kappa^{2}$ is the constant of gravitation, $M_{\mathrm{S}}$ the mass of the Sun, and $r$ is distance between the asteroid and the Sun centers of mass. Here, $P_{2}^{0}(\sin \delta)$ and $P_{2}^{2}(\sin \delta)$ are two associated Legendre polynomials, $\alpha$ and $\delta$ represent the body-centered longitude and latitude of the Sun, respectively, both expressed with respect to the equatorial plane of the asteroid.

We focus on the first term of the potential on the righthand side of Eq. (5), which depends on the oblatness of the asteroid. The second term depends on the triaxiality of the asteroid and has been studied in detail by Souchay \& Bouquillon (2005) in the case of (433) Eros. They show that its contribution to the motion of nutation gives high frequency variations, corresponding roughly to half of the period of the rotation and in consequence generates after integration terms with very small amplitudes. Thus we can ignore the effect of the triaxiality, i.e. the second component on the righthand side of Eq. (5), at the first order of our calculations.

Then it is useful to express $P_{2}^{0}(\sin \delta)$ as a function of $\lambda$ and $\beta$, which are the relative latitude and longitude of the Sun with respect the reference plane. Kinoshita (1977) gives us this transformation, starting from Jacobi polynomials. He showed that $P_{2}(\sin \delta)$ can be divided into three components, directly depending on the factors $\frac{1}{2}\left(3 \cos ^{2} J-1\right), \sin 2 J$, and $\sin ^{2} J$, where $J$ stands for the angular distance between the axis of figure and the axis of angular momentum. As already set as a hypothesis, these two axes are very close, so that we can consider $J \approx 0$. Thus we take only the first term into account depending on $\frac{1}{2}\left(3 \cos ^{2} J-1\right) \approx 1$ and we have

$$
\begin{aligned}
P_{2}^{0}(\sin \delta)= & \frac{1}{2}\left(3 \cos ^{2} J-1\right)\left[\frac{1}{2}\left(3 \cos ^{2} I-1\right) P_{2}(\sin \beta)\right. \\
& -\frac{1}{2} \sin [2 I] P_{2}^{1}(\sin \beta) \sin [\lambda-h] \\
& \left.-\frac{1}{4} \sin ^{2}[I] P_{2}^{2}(\sin \beta) \cos [2 \lambda-2 h]\right]
\end{aligned}
$$

where: $I=-\epsilon$ is the obliquity.

Moreover, we call that we have chosen as the basic reference plane the orbital plane of the asteroid at the basic epoch J2000.0. On short time scales, the orbital plane does not move significantly, and we can adopt the value $\beta \approx 0$ in our calculations.

Finally the expression of the perturbing solar potential takes the simplified form:

$$
U_{\text {sun }}^{*} \approx k \times\left[\frac{a}{r}\right]^{3} \times\left[-\frac{1}{4}\left(3 \cos I^{2}-1\right)-\frac{3}{4} \sin I^{2} \cos [2 \lambda-2 h]\right] .
$$

Where, using using Kepler's third law:

$k=\frac{\kappa^{2} M_{\mathrm{s}}}{a^{3}} \times \frac{2 C-A-B}{2}=n^{2} \times \frac{2 C-A-B}{2}$

with $a$ the semi-major axis and $n$ the mean motion of the asteroid.

\subsection{Development of the equations as functions of the mean anomaly}

If we introduce this simplified expression of the potential in Eqs. (4), we have

$\begin{aligned} \Delta \psi & =\frac{K}{2} \cos I \times \int\left[\left(\frac{a}{r}\right)^{3}-\left(\frac{a}{r}\right)^{3} \cos [2 \lambda-2 h]\right]_{\mathrm{per}} \mathrm{d} t \\ \Delta \epsilon & =-\frac{K}{2} \sin I \times \int\left[\left(\frac{a}{r}\right)^{3} \sin [2 \lambda-2 h]\right]_{\mathrm{per}} \mathrm{d} t .\end{aligned}$

Here we include the scaling factor $K$ (Kinoshita 1977):

$K=\frac{3 n^{2}}{\omega_{a}} \times \frac{2 C-A-B}{2 C}=\frac{3 n^{2}}{\omega_{a}} \times H_{\mathrm{d}}$,

with:

$H_{\mathrm{d}}=\frac{C-(A+B) / 2}{C}$,

where $H_{\mathrm{d}}$ represents the dynamical ellipticity of the asteroid, characterizing its flattening. The scaling factor $K$ gives the magnitude of the amplitude of the preceession-nutation motion, and we see that it is proportional to the square of the mean motion $n$, to the dynamical ellipticity $H_{\mathrm{d}}$, and to the inverse of the rotation rate $\omega_{a}$. As the obliquity, $K$ is a crucial parameter that we report in our results in Sect. 6.

As shown in Souchay et al. (2003) in the case of (433) Eros, we can develop Eqs. (22) at any order of eccentricity $e$ and as a function of the sole mean anomaly $M$ of the asteroid. The longitude is counted from $\gamma_{\mathrm{J} 2000}$, the fixed vernal point of the asteroid: i.e., the ascending node of the orbital plane referred to the equatorial plane of the epoch J2000.0. Thus, the longitude can be split as $\lambda=\omega+v+\gamma N$ (see Fig. 2) where $\gamma N$ is the angle between $\gamma$, and the ascending node $N$ of the orbital plane refers to the ecliptic of $\mathbf{J} 2000, \omega$ and $v$ are respectively the argument of the periapsis and the true anomaly. But as we see in Sect. 4.3, we initially have access to $\gamma^{\prime}$, the descending node, which is 180 degree opposite to $\gamma$. Thus we finally we write $\lambda=\omega+\nu+180^{\circ}-\gamma^{\prime} N$. We note that $\Lambda=\gamma^{\prime} N$. We explain how to calculate this angle in the Sect. 4.3.

We can split the different terms of Eqs. (22) as

$$
\left(\frac{a}{r}\right)^{3} \cos [2(\lambda-h)]=C^{\prime}\left(\frac{a}{r}\right)^{3} \cos [2 v]-S^{\prime}\left(\frac{a}{r}\right)^{3} \sin [2 v]
$$

and

$\left(\frac{a}{r}\right)^{3} \sin [2(\lambda-h)]=S^{\prime}\left(\frac{a}{r}\right)^{3} \cos [2 v]+C^{\prime}\left(\frac{a}{r}\right)^{3} \sin [2 v]$,

where $C^{\prime}=\cos [2(\omega-\Lambda-h)]$ and $S^{\prime}=\sin [2(\omega-\Lambda-h)]$ can be considered as two constants for the interval of time that we consider, because of the very low rate of change of the argument of the periapsis $\omega$, the angle $\Lambda$ defined above and the precession angle $h$ involved. Moreover, we assume that $h=-\psi$ is a small variable. 


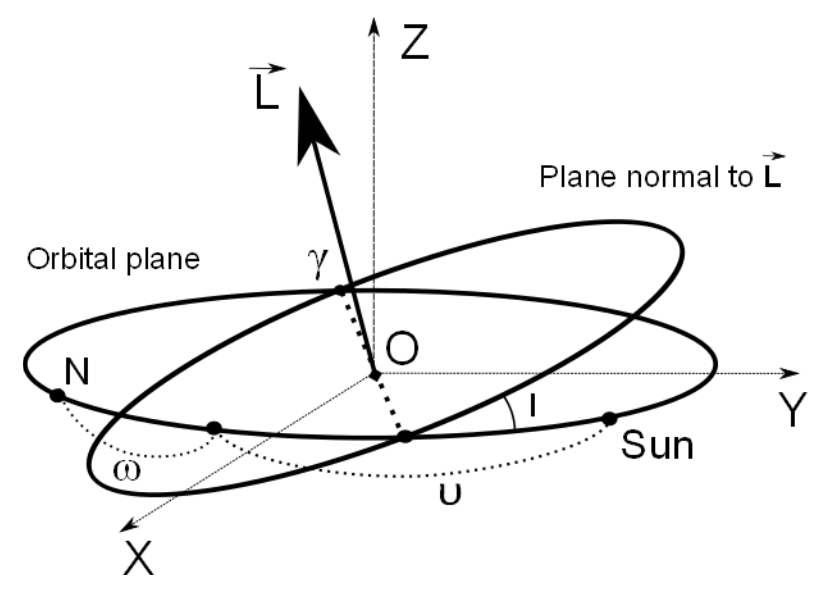

Fig. 2. Orbital plane and the plane normal to the angular momentum vector. The Sun longitude $\lambda$ is counted along the orbital plane from the vernal point $\gamma$.

Souchay et al. (2003a) give the general developments of the three terms function of $M$ and $v$, i.e. $\left[\frac{a}{r}\right]^{3},\left[\frac{a}{r}\right]^{3} \cos (2 v)$, and $\left[\frac{a}{r}\right]^{3} \sin (2 v)$. Here, we can use the Cayley coefficients to expand functions of the true anomaly in Fourier series of the mean anomaly from the following expressions (Hughes 1981):

$\left(\frac{r}{a}\right)^{i} \cos [j v]=\sum_{k} C_{k}^{i, j}[e] \cos [k M]$
$\left(\frac{r}{a}\right)^{i} \sin [j v]=\sum_{k} S_{k}^{i, j}[e] \sin [k M]$.

Thus we have

$$
\begin{aligned}
\left(\frac{a}{r}\right)^{3} & =\sum_{k} C_{k}^{-3,0}[e] \cos [k M] \\
& =\left(1+\frac{3 e^{2}}{2}+\ldots\right)+\left(3 e+\frac{27 e^{3}}{8}+\ldots\right) \cos [M]+\ldots
\end{aligned}
$$

$\left(\frac{a}{r}\right)^{3} \cos [2 v]=\sum_{k} C_{k}^{-3,2}[e] \cos [k M]$

$$
\begin{aligned}
= & \left(-\frac{e}{2}+\frac{e^{3}}{12}+\ldots\right) \cos [M] \\
& +\left(1-\frac{5 e^{2}}{2}+\frac{41 e^{4}}{48}+\ldots\right) \cos [2 M]+\ldots
\end{aligned}
$$

$$
\begin{aligned}
\left(\frac{a}{r}\right)^{3} \sin [2 v] & =\sum_{k} S_{k}^{-3,2}[e] \sin [k M] \\
& =\left(-\frac{e}{2}+\frac{e^{3}}{24}+\ldots\right) \sin [M] \\
& +\left(1-\frac{5 e^{2}}{2}+\frac{37 e^{4}}{48}+\ldots\right) \sin [2 M]+\ldots
\end{aligned}
$$

Following their way to calculate we are able to express the equations of motion as function of cosines and sinus of the mean anomaly $M$.

\subsection{The nutation coefficients}

After expressing the Eqs. (9a) and (9b) with the Cayley coefficients, we use the expression of the mean motion $n=\frac{\mathrm{d} M}{\mathrm{~d} t}$ to integrate. First, for the motion in longitude,

$$
\begin{aligned}
\Delta \psi= & \frac{K}{2} \cos I \times \int\left[\left(\frac{a}{r}\right)^{3}-\left(\frac{a}{r}\right)^{3} \cos [2 \lambda-2 h]\right]_{\mathrm{per}} \mathrm{d} t \\
= & \frac{K}{2} \cos I \times \int\left[\left(\frac{a}{r}\right)^{3}\left(1-C^{\prime} \cos [2 v]+S^{\prime} \sin [2 v]\right)\right]_{\mathrm{per}} \mathrm{d} t \\
= & \frac{K}{2} \cos I \int\left[\sum_{k} C_{k}^{-3,0}[e] \cos [k m]-C^{\prime} \sum_{k} C_{k}^{-3,2}[e] \cos [k M]\right. \\
& \left.+S^{\prime} \sum_{k} S_{k}^{-3,2}[e] \sin [k M]\right]_{\mathrm{per}} \frac{\mathrm{d} M}{n}
\end{aligned}
$$

And after the integration,

$$
\begin{aligned}
\Delta \psi= & \frac{K}{2} \cos I\left[\sum_{k} C_{k}^{-3,0}[e] \frac{\sin [k m]}{k n}-C^{\prime} \sum_{k} C_{k}^{-3,2}[e] \frac{\sin [k M]}{k n}\right. \\
& \left.-S^{\prime} \sum_{k} S_{k}^{-3,2}[e] \frac{\cos [k M]}{k n}\right]_{\mathrm{per}}
\end{aligned}
$$

And similarly for the motion in obliquity,

$$
\begin{aligned}
\Delta \epsilon= & -\frac{K}{2} \sin I \times \int\left[\left(\frac{a}{r}\right)^{3} \sin [2 \lambda-2 h]\right]_{\mathrm{per}} \mathrm{d} t \\
= & -\frac{K}{2} \sin I \times \int\left[\left(\frac{a}{r}\right)^{3}\left(S^{\prime} \cos [2 v]+C^{\prime} \sin [2 v]\right)\right]_{\mathrm{per}} \mathrm{d} t \\
= & -\frac{K}{2} \sin I \times \int\left[S^{\prime} \sum_{k} C_{k}^{-3,2}[e] \cos [k m]\right. \\
& \left.+C^{\prime} \sum_{k} S_{k}^{-3,2}[e] \sin [k M]\right]_{\mathrm{per}} \frac{\mathrm{d} M}{n}
\end{aligned}
$$

Finally,

$$
\begin{aligned}
\Delta \epsilon= & -\frac{K}{2} \sin I \times\left[S^{\prime} \sum_{k} C_{k}^{-3,2}[e] \frac{\sin [k M]}{k n}\right. \\
& \left.-C^{\prime} \sum_{k} S_{k}^{-3,2}[e] \frac{\cos [k M]}{k n}\right]_{\mathrm{per}} .
\end{aligned}
$$

The last step consists in developing the Cayley coefficients to the fourth order of e. The eccentricity of the bodies concerned is never greater than 0.28 . Thus, it seems enough to stop our calculations at the fifth order of e, which gives us terms of amplitude lower than 2 mas. In fact, the uncertainties on precession and nutation motion do not depend on theoretical uncertainties or on orbital parameters, which are very well known at the sixth or seventh digit, but especially of observational variables $H_{\mathrm{d}}$ and $\epsilon$. 
By keeping only the periodic terms, we obtain the analytical expression of the nutation in longitude $\Delta \psi$ and in obliquity $\Delta \epsilon$ :

$$
\begin{aligned}
\Delta \psi= & \frac{K}{2} \cos I \times\left[\left(3 e+\frac{27}{8} e^{3}+C^{\prime}\left[\frac{e}{2}-\frac{e^{3}}{12}\right]\right) \frac{\sin M}{n}\right. \\
& +\left(\frac{9}{2} e^{2}+\frac{7}{2} e^{4}+C^{\prime}\left[-1+\frac{5}{2} e^{2}-\frac{41}{48} e^{4}\right]\right) \frac{\sin 2 M}{n} \\
& +\left(\frac{53}{8} e^{3}+C^{\prime}\left[-\frac{7}{2} e+\frac{123}{16} e^{3}\right]\right) \frac{\sin 3 M}{3 n} \\
& +\left(\frac{77}{8} e^{4}+C^{\prime}\left[-\frac{17}{2} e^{2}+\frac{115}{6} e^{4}\right]\right) \frac{\sin 4 M}{4 n} \\
& -C^{\prime} \frac{845}{48} e^{3} \frac{\sin 5 M}{5 n}-C^{\prime} \frac{533}{16} e^{4} \frac{\sin 6 M}{6 n} \\
& -S^{\prime}\left(-\frac{e}{2}+\frac{e^{3}}{24}\right) \frac{\cos M}{n} \\
& -S^{\prime}\left(1-\frac{5}{2} e^{2}+\frac{37}{48} e^{4}\right) \frac{\cos 2 M}{n} \\
& -S^{\prime}\left(\frac{7}{2} e-\frac{123}{16} e^{3}\right) \frac{\cos 3 M}{3 n} \\
& -S^{\prime}\left(\frac{17}{2} e^{2}-\frac{115}{6} e^{4}\right) \frac{\cos 4 M}{4 n} \\
& \left.-S^{\prime} \frac{845}{48} e^{3} \frac{\cos 5 M}{5 n}-S^{\prime} \frac{533}{16} e^{4} \frac{\cos 6 M}{6 n}\right]
\end{aligned}
$$$$
\Delta \epsilon=-\frac{K}{2} \sin I \times\left[-\left(-\frac{e}{2}+\frac{e^{3}}{24}\right) \frac{C^{\prime} \cos M}{n}\right.
$$$$
+\left(-\frac{e}{2}+\frac{e^{3}}{12}\right) \frac{S^{\prime} \sin M}{n}
$$$$
+\left(-1+\frac{5}{2} e^{2}-\frac{37}{48} e^{4}\right) \frac{C^{\prime} \cos 2 M}{2 n}
$$$$
+\left(1-\frac{5}{2} e^{2}+\frac{41}{48} e^{4}\right) \frac{S^{\prime} \sin 2 M}{2 n}
$$$$
-\left(\frac{7}{2} e-\frac{123}{16} e^{3}\right) \times \frac{C^{\prime} \cos 3 M-S \sin 3 M}{3 n}
$$$$
-\left(\frac{17}{2} e^{2}-\frac{115}{6} e^{4}\right) \times \frac{C^{\prime} \cos 4 M-S \sin 4 M}{4 n}
$$$$
-\frac{845}{48} e^{3} \times \frac{C^{\prime} \cos 5 M-S^{\prime} \sin 5 M}{5 n}
$$$$
\left.-\frac{533}{16} e^{4} \times \frac{C^{\prime} \cos 6 M-S^{\prime} \sin 6 M}{6 n}\right] \text {. }
$$

\subsection{The precession rate}

To give the expression of the precession rate, we start from the formula (20), since for the nutation we develop the Cayley coefficients but only keep the secular part. We have the following development to the fourth order of $e$ :

$\dot{\psi} \approx \frac{K}{2} \cos I\left[1+\frac{3}{2} e^{2}+\frac{15}{8} e^{4}\right]$

where $K$ is given by (10).

\section{Initial conditions}

To compute the precession rates, as well as the nutation coefficients, according to the expressions above we need to know the orbital dynamics of the asteroid but also its spin axis orientation, its spin rate, and its moments of inertia.

\subsection{Orbital dynamics}

To carry out our calculations, we need the set of orbital parameters $(a, e, i, \omega, \Omega, M)$ of the asteroid. At first approximation, we can set the five first parameters as constants for the short time scale considered in our investigations, typically a few decades or a hundred years. These orbital parameters are given by JPL's HORIZONS system ${ }^{1}$. The orbital parameters $e$ and $M$ are explicitly involved in Eqs. (23) and (24), whereas the value of $a$ is needed to compute the constant $K$ according to (10). At last $i, \omega$, and $\Omega$ will be used for the orientation of the spin axis (see next section).

\subsection{The spin characteristics}

At a given date, the spin characteristics of an asteroid are essentially given by two parameters: the obliquity, i.e. the angle between the equatorial and the orbital planes of the object, and its spin angular rate. The equations of the rotational motion given by formula, (23), (24), and (25) depend directly on the spin angular rate $\omega_{r}$, through the presence of $K$, and on the obliquity $\epsilon=-I$. Despite its fundamental physical importance, for instance, in terms of seasons, climate, and solar flux, in particular, the obliquities of the asteroids are rarely given in the literature. As a consequence we need to calculate it for our set of asteroids.

For at purpose we use two unit vectors. First the vector normal to the orbital plane, defined by the orbital elements $\Omega$ and $i$ with respect to the mean equinox and ecliptic of J2000.0:

$z=\left(\begin{array}{c}\sin i \sin \Omega \\ \cos \Omega \sin i \\ \cos i\end{array}\right)$

and second, the vector linked to the axis of figure that is given in terms of its right ascension $\alpha_{f}$ and its declination $\delta_{f}$ with respect to the ICRF. We make a basic and trivial transformation to get its longitude $\lambda_{f}$ and latitude $\beta_{f}$, with respect to the mean equinox and ecliptic J2000.0 (see for instance Bills \& Nimmo 2011):

$\boldsymbol{f}=\left(\begin{array}{c}\cos \beta_{f} \cos \lambda_{f} \\ -\cos \beta_{f} \sin \lambda_{f} \\ \sin \beta_{f}\end{array}\right)$.

The obliquity $\epsilon$ is then obtained by inverting the equations of the scalar product and of the cross product of these two vectors from one of the following equations:

$z \cdot f=\cos \epsilon, \quad z \times f=w \sin \epsilon$.

\subsection{Calculation of the longitude}

We let $\gamma$ and $\gamma^{\prime}$ be, respectively, the ascending and descending nodes of the asteroid orbital plane with respect to its equatorial plane. Let $N$ be the ascending node of the orbital plane with respect to the reference plane, i.e. the ecliptic of J2000.0. In Eqs. (22) involving the longitude $\lambda$, it is counted from $\gamma$ as following $\lambda=\omega+v+180^{\circ}-\Lambda$ where $\Lambda=\gamma^{\prime} N$. To calculate this last angle, which appears as a constant in the equations of motion, we point out that $w$, colinear to the cross product of unit vectors $z$ and $\boldsymbol{f}$, is directed toward $\gamma^{\prime}$ (see Fig. 3).

Thus, we get

$\Lambda=\gamma^{\prime} N=\arccos (\boldsymbol{O N} \cdot \boldsymbol{w})$

and can determine $\lambda$ starting from this determination of $\Lambda$.

1 http://ssd.jpl.nasa.gov/ 


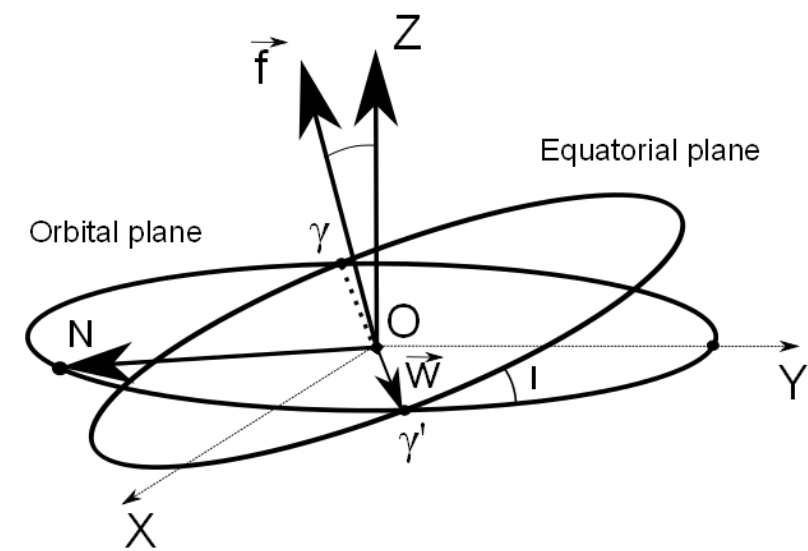

Fig. 3. Vector $f$ normal to the equatorial plane and to the orbital plane.

\subsection{Moments of inertia}

To constrain the rotational dynamical equations of each asteroid in a satisfying manner, we need to rather accurately know its moments of inertia, which appear directly in the factor $K$. Thanks to space missions or accurate ground-based observations of light curves, we can have access to a well defined shape and spin-pole orientation of the asteroids. This is the case of the five asteroids involved in our study. As mentioned before, we assume here that we can approximate their shape as that of a triaxial ellipsoid with $a, b$, and $c$ as axial dimensions $(c<b<a)$. If the density of the asteroid is supposed to be uniform, we can deduce its moments of inertia directly (Bills \& Nimmo 2011):

$\left(\begin{array}{l}A \\ B \\ C\end{array}\right)=\frac{M}{5}\left(\begin{array}{l}b^{2}+c^{2} \\ a^{2}+c^{2} \\ a^{2}+b^{2}\end{array}\right)$

In case the body is differentiated, which should be the case of minor planets such as (1) Ceres or (4) Vesta, we need to make some assumptions about the internal structure to calculate the moments of inertia. Moreover, in case a spacecraft encouters the asteroid closely enough, it should be possible to determine the low-degree gravitational potentials $J_{2}=-C_{2,0}$ and $C_{2,2}$. If we have an estimation of the polar moment of inertia $C$, we can calculate the normalized moments of inertia $\bar{A}$ and $\bar{B}$ with the following formula (Konopliv 2011):

$\left\{\begin{array}{l}\bar{A}=\frac{C}{M R^{2}}-J_{2}-2 C_{2,2} \\ \bar{B}=\frac{C}{M R^{2}}-J_{2}+2 C_{2,2},\end{array}\right.$

where $M$ and $R$ stand for the mass and mean radius of the body, respectively. The problem remains to determine the largest moment of inertia $\bar{C}$. For that purpose Konopliv et al. (2011) present several solutions. If we have an estimation of $J_{2}$ and if we assume the hydrostatic equilibrium, then we can use the Radau-Darwin relation (32),

$\bar{C}=\frac{C}{M R^{2}}=\frac{2}{3}\left(1-\frac{2}{5} \sqrt{\frac{4-k}{1+k}}\right)$,

where $k$ is a secular Love number that can be deduced from $J_{2}$. Another solution consists in establishing a model of the internal structure, using in situ observations or based on simulations of the evolution of the body.

\section{Physical parameters}

As seen in the previous sections, to calculate the precession rate and nutation coefficients of an asteroid, in addition of the orbital elements, we need to know the spin axis orientation, the rotational angular rate, and the moments of inertia. In this paper we have selected five well constrained asteroids for an accurate determination of their precession-nutation motion. Four of them have recently been visited by spacecrafts, and in consequence, their physical and rotational parameters have been accurately established. These asteroids are (433) Eros, (2867) Steins, (25143) Itokawa, and (4) Vesta. The additional asteroid studied here is (1) Ceres, which will be oberved by DAWN in 2015.

In the following, we present the most accurate data that we have found in the literature but also the methods by which they were obtained. The data used for our calculations will be summarized in Table 3.

\subsection{Parameters for (433) Eros, (2867) Steins, and (25143) Itokawa}

In the case of (433) Eros, the data obtained by the NEAR probe was analyzed by Miller et al. (2002), who could determine the spin vector orientation with remarkable accuracy better than 0.001 degree $\left(\approx 4^{\prime \prime}\right)$ and a rotational period with eight digits accuracy $T=5.27025547 \mathrm{~h}$. From the spin vector orientation given in equatorial coordinates with the origin at the center of the asteroid, Souchay et al. (2003a) could deduce a remarkable value of the obliquity: $\epsilon=89^{\circ}$. This means that like Uranus, Eros' spin axis is nearly oriented in the orbital plane. Eros' moments of inertia were solved with high accuracy by integrating the shape model and assuming uniform density. Errors were minimized with the help of gravity harmonics determined by tracking of the orbit spacecraft, which shows a very high correlation with the shape model, sustaining the idea of a very homogeneous body. In the case of this asteroid, the normalized moment of inertia $\bar{A}=17.09 \mathrm{~km}^{2}$ is considerably smaller than the two other ones $\bar{B}=71.79 \mathrm{~km}^{2}$ and $\bar{C}=74.49 \mathrm{~km}^{2}$ (Miller et al. 2002), which indicates a high value of the triaxiality. For this reason the effects of the triaxiality on nutation were studied in a specific paper by Souchay et al. (2003b). As already mentioned before, their conclusion is that despite its high value, Eros' triaxiality generates relatively small components, which can be neglected at first order with respect to nutation coefficients due to the flattening (Souchay et al. 2003; Souchay \& Bouquillon 2005).

Demura et al. (2006) analyzed the data of the Hayabusa mission derived from the AMICA (Asteroid Multiband Imaging CAmera) to determine the locations of the pole and rotation axis of (25143) Itokawa. The positioning of the latter, established in equatorial coordinates, is given with $\mathrm{a} \pm 3.9^{\circ}$ margin and characterizes a retrograd rotation, with a rotation axis almost perpendicular to the ecliptic plane. The rotation period of Itokawa was determined at the fith digit: $T=12.132 \mathrm{~h}$, whereas the size of the three rectangular axes were set to $535 \times 298 \times 244 \mathrm{~m}$ with $1-\mathrm{m}$ uncertainty. Assuming a homgenous density inside the asteroid and a trixial shape, it is possible to deduce the moments of inertia and $H_{\mathrm{d}}$ fairly precisely.

Jorda et al. (2012) have implemented several threedimensional reconstruction techniques from the onboard OSIRIS camera to retrieve the global shape of (2867) Steins. They could reconstruct an approximate shape model, showing that Steins looks like a ellipsoid with principal axes $6.83 \times$ $5.70 \times 4.42 \mathrm{~km}$. As in the case of (25143) Itokawa, we could deduce moments of inertia from the Eq. (30) from these values. 
Table 1. Moments of inertia of (4) Vesta according to two bibliographic sources.

\begin{tabular}{lccc}
\hline \hline Authors & $A$ & $B$ & $C$ \\
\hline Bills \& Nimmo (2011) & 0.3610 & 0.3858 & 0.4532 \\
Rambaux (2013) & 0.3433 & 0.3546 & 0.42 \\
\hline
\end{tabular}

The same authors also determined the equatorial coordinates of the polar axis, implying a very large obliquity of $172^{\circ}$ and indicating a retrograd motion. Nevertheless, their accuracy for the spin axis orientation is only given with an uncertainty of $\pm 5^{\circ}$.

\subsection{Parameters for (4) Vesta}

As the second most massive body of the main asteroid belt, (4) Vesta has been visited during the DAWN mission from July 2011 to August 2012. The tracking of the spacecraft gives us a good accurate determination of the low-degree gravity field, so that we can make an assumption about the internal structure of the asteroid. Data suggest that (4) Vesta has an iron core, a mantle, and crust (Asmar et al. 2012). In Table 1, we report the values of the moments of inertia as deduced from two studies (Bills \& Nimmo 2011; Rambaux 2013). For our study, we chose the values proposed by these last authors. To study the rotational motion of (4) Vesta, they used a two-layer model of internal structure (Asmar et al. 2012), from which they provide the polar moment of inertia and deduce the two other ones with formula (31). We notice that Asmar et al. (2012) also give us the spin pole orientation with a very good accuracy if we compare it to those of other asteroids. Indeed the right ascension and declination of the north polar axis are $\alpha=309^{\circ} .031 \pm 0.003$ and $\delta=42.2264^{\circ} \pm 0.0002$, respectively.

\subsection{Parameters for (1) Ceres}

Qualified as a minor planet, (1) Ceres, is the most massive object of the asteroid belt, accounting for one third of the main asteroid belt mass. The spacecraft DAWN should normally be placed in orbit around it in 2015. As for (4) Vesta, we expect precious data about its internal structure (McCord et al. 2011). However, from ground-based observations and the Hubble Space Telescope, its shape is well defined, and we can already assume some solid hypotheses: with a mean radius of $467.6 \pm 2.2 \mathrm{~km}$, (1) Ceres has a spheroid shape with a small oblateness as terrestrial planets (Carry et al. 2008). Recent data indicate that (1) Ceres has undergone a thermal evolution and that we can suppose a differentiated model of the internal structure (Castillo-Rogez \& McCord 2010). Because of these new observations, it is more appropriate to call it a protoplanet in comparison with with other asteroids. From these assertions, Rambaux (2013) studied the free rotational motion of (1) Ceres after calculating its moments of inertia. Carry et al. (2008) provide us the spin pole orientation with a precision of $\pm 5^{\circ}$, and Chamberlain et al. (2007) furnish the rotational period with six-digit accuracy: $T=$ $9.074170 \mathrm{~h} \pm 0.000002$.

For different models of internal structure, McCord \& Sautin (2005) give a range of possible values of the largest moment of intertia $C$. With an estimation of the shape, Bills \& Nimmo (2011) and Lara et al. (2011) have calculated the values of the three moments of inertia with the formula (30). At last, using a dedicated model of internal structure, Rambaux et al. (2011) calculated the mean moment of inertia and the three principal moments $A, B$, and $C$. All these results are presented in Table 2 .
Table 2. Moments of inertia of (1) Ceres, according to four bibliographic sources.

\begin{tabular}{lccc}
\hline \hline Authors & $A$ & $B$ & $C$ \\
\hline McCord \& Sautin (2005) & - & - & 0.304 to 0.4 \\
Bills \& Nimmo (2011) & 0.3899 & 0.3899 & 0.4202 \\
Lara et al. (2011) & 0.39096 & 0.39105 & 0.41799 \\
Rambaux et al. (2011) & 0.347 & 0.3394 & 0.3623 \\
\hline
\end{tabular}

Therefore, even though (1) Ceres has not yet been explored by a space mission, that data looks precise enough to give an accurate modeling of its rotational motion.

\section{Results}

\subsection{Precession-nutation characteristics}

In the previous sections we have presented an analytical model for calculating the rotational motion of any of the asteroids that were considered here. Thanks to this model and accurate data provided either by up-to-date ground-based observations (for (1) Ceres) or by recent space missions (for the other four asteroids), we present the three fundamental parameters that are necessary for this calculation in Table 4 . In particular, we show the values for each asteroid of our determinations for the obliquity $\epsilon=-I$ and the dynamical ellipticiy $H_{\mathrm{d}}$. We call that $H_{\mathrm{d}}$ characterizes the flattening of the body, which is not established from its axes lengths but starting from its moments of inertia $A$, $B, C$. More precisely,

$H_{\mathrm{d}}=\frac{C-(A+B) / 2}{C}$

In addition we give the scaling factor $K$, which is directly proportional to $H_{\mathrm{d}}$, to the mean motion $n$, and to the inverse of the spin angular velocity $\omega_{a}$, according to (9). For the asteroids (433) Eros and (25143) Itokawa, both characterized by a very irregular shape, the scaling factor $K$ is particularly big, with values 22.3 times and 65.0 times greater than for (1) Ceres. Then we could expect for these two cases a high value of the nutation coefficients and of the precession rate.

Finally we report in Table 4 the precession rate calculated by the formula (25), as well as the amplitudes of the leading nutation coefficients in longitude and obliquity, respectively, as calculated from the formulas (23) and (24). We used the same IAU conventions for the sign of the precession as for the Earth, which means that a negative value of $\dot{\psi}$ corresponds to a prograde motion. This is the case of (25143) Itokawa and (2867) Steins, in contrast to the three other asteroids showing a retrograde precession motion as for our planet.

In the case of (433) Eros, because of the coefficient $\cos \epsilon$ at the righthand side of the formula (25), with $\epsilon=89^{\circ}$, the value of the precession rate becomes in fact very low. On the opposite, we also notice that for (25143) Itokawa, the precession motion with a value of $\dot{\psi}=-(461.52 \pm 6.57)^{\prime \prime} / \mathrm{yr}$, reaches more than one degree in eight years. By comparison, we recall that the precession rate of the Earth owing to the sole effect of the Sun (as it is determined here) participates roughly one third of the lunisolar precession rate, that is to say, $15^{\prime \prime} / \mathrm{cy}$. Therefore the ratio for Itokawa roughly corresponds to 30 times this value. Thus, we could expect an observational detection of the modification of the spin orientation of (25143) Itokawa over a period of a few decades, owing to the precession, which has still never been done for any asteroid. This should be all the more possible since the synthetic 
Table 3. Physical parameters used for the rotational study of the five selected asteroids.

\begin{tabular}{lccccc}
\hline \hline Asteroid & Shape $(\mathrm{km})$ & Spin-rate $(\mathrm{h})$ & RA(J2000) $(\mathrm{deg})$ & Dec $(\mathrm{J} 2000)(\mathrm{deg})$ & Moments of intertia $\left(\mathrm{km}^{2}\right)$ \\
\hline (433) Eros & $36 \times 15 \times 13$ & 5.27025547 & 11.3692 & 17.2273 & $17.09 \times 71.79 \times 74.49$ \\
(2867) Steins & $6.83 \times 5.70 \times 4.42$ & 6.049 & 99 & -59 & $26.01 \times 33.09 \times 39.57$ \\
(25143) Itokawa & $535 \times 298 \times 244 \mathrm{~m}$ & 12.132 & 128.5 & -89.66 & $0.06 \times 0.16 \times 0.19$ \\
(4) Vesta & $282 \times 267 \times 221$ & 5.3421 & 309.031 & 42.2264 & $0.3433 \times 0.3546 \times 0.42$ \\
(1) Ceres & $479.7 \times 479.7 \times 444.4$ & 9.07410 & 288 & 66 & $0.3394 \times 0.3394 \times 0.3623$ \\
\hline
\end{tabular}

Table 4. Parameters of the rotational motion.

\begin{tabular}{lcccccccc}
\hline \hline Asteroid & Obliquity $\left(^{\circ}\right)$ & $H_{\mathrm{d}}$ & $K\left[^{\prime \prime} / \mathrm{y}\right]$ & $\dot{\psi}\left[{ }^{\prime \prime} / \mathrm{y}\right]$ & $\Delta \psi\left[{ }^{\prime \prime}\right]$ & $\Delta \epsilon\left[^{\prime \prime}\right]$ & $R_{m} \Delta \psi[\mathrm{m}]$ & $R_{m} \Delta \epsilon[\mathrm{m}]$ \\
\hline (433) Eros & 89.00 & 0.4034 & 304.0953 & 2.8456 & 0.7437 & 27.3351 & $3.3300 .10^{-2}$ & 1.2240 \\
(2867) Steins & 161.84 & 0.2717 & 55.3000 & -27.1470 & 13.8229 & 2.8589 & $1.7759 .10^{-1}$ & $3.6730 .10^{-2}$ \\
(25143) Itokawa & 157.30 & 0.3828 & 885.7308 & -461.5208 & 138.7273 & 28.2561 & $1.0761 .10^{-1}$ & $2.1918 .10^{-2}$ \\
(4) Vesta & 27.46 & 0.1692 & 30.4449 & 13.6728 & 5.1460 & 2.2122 & 6.5540 & 2.8175 \\
(1) Ceres & 3.91 & $7.1652 .10^{-2}$ & 13.6224 & 6.8584 & 3.1834 & 0.1813 & 7.2169 & 0.4150 \\
\hline
\end{tabular}

Table 5. Nutation in longitude for (4) Vesta.

\begin{tabular}{lcccc}
\hline \hline Sin $\left({ }^{\prime \prime}\right)$ & Cos $\left({ }^{\prime \prime}\right)$ & Amp $\left({ }^{\prime \prime}\right)$ & Period (year) & Arg \\
\hline 2.3787 & 0.2431 & 1.1027 & 3.6287 & $\mathrm{M}$ \\
-2.6121 & -2.6481 & 1.7154 & 1.8143 & $2 \mathrm{M}$ \\
-0.5676 & -0.5576 & 0.3669 & 1.2096 & $3 \mathrm{M}$ \\
-0.0939 & -0.0914 & 0.0604 & 0.9072 & $4 \mathrm{M}$ \\
-0.0144 & -0.0139 & 0.0092 & 0.7257 & $5 \mathrm{M}$ \\
-0.0021 & -0.0020 & 0.0013 & 0.6048 & $6 \mathrm{M}$ \\
\hline
\end{tabular}

light curves for Itokawa are a priori very well constrained thanks to the data concerning the shape and albedo, obtained from the Hayabusa mission.

In Table 4, it is also interesting to point out that (4) Vesta has an obliquity that is almost identical to that of the Earth $\left(27^{\circ} .46\right.$ instead of 23.45) and that the amplitude of the precession rate is very close when compared with the part due to the solar effect for our planet already mentioned above, i.e., $\approx 15^{\prime \prime} / \mathrm{y}$. In the last columns, we have reported the amplitude of the real nutation amplitude of the asteroids in space, by scaling it to the size of the asteroid. Only (1) Ceres and (4) Vesta present a significant surface displacement of several meters, owing to their relatively big size.

In Fig. 4 (top), we plot as a function of the time the combined precession-nutation motion in longitude $\psi+\Delta \psi$ of (25143) Itokawa and (2867) Steins, for which this motion is by far more important than for the three other bodies considered. In Fig. 4 (bottom), we present the corresponding plot for our set of five asteroids excepted (25143) Itokawa, for which the motion is drastically more important than any other one, and cannot be put on the same scale.

\subsection{The nutation motion}

Thanks to Eqs. (23) and (24), we can directly present the values of the coefficients of nutation respectively in longitude and in obliquity one by one. Thus for (4) Vesta, we present the tables of nutation both in longitude (Table 5) and in obliquity (Table 6) in detail as a series of sinusoidal components with arguments that are multiples of the mean anomaly $M$ of the asteroid. We can see that the semi-annual term dominates, with a secondary annual component comparatively much bigger in longitude than in obliquity.
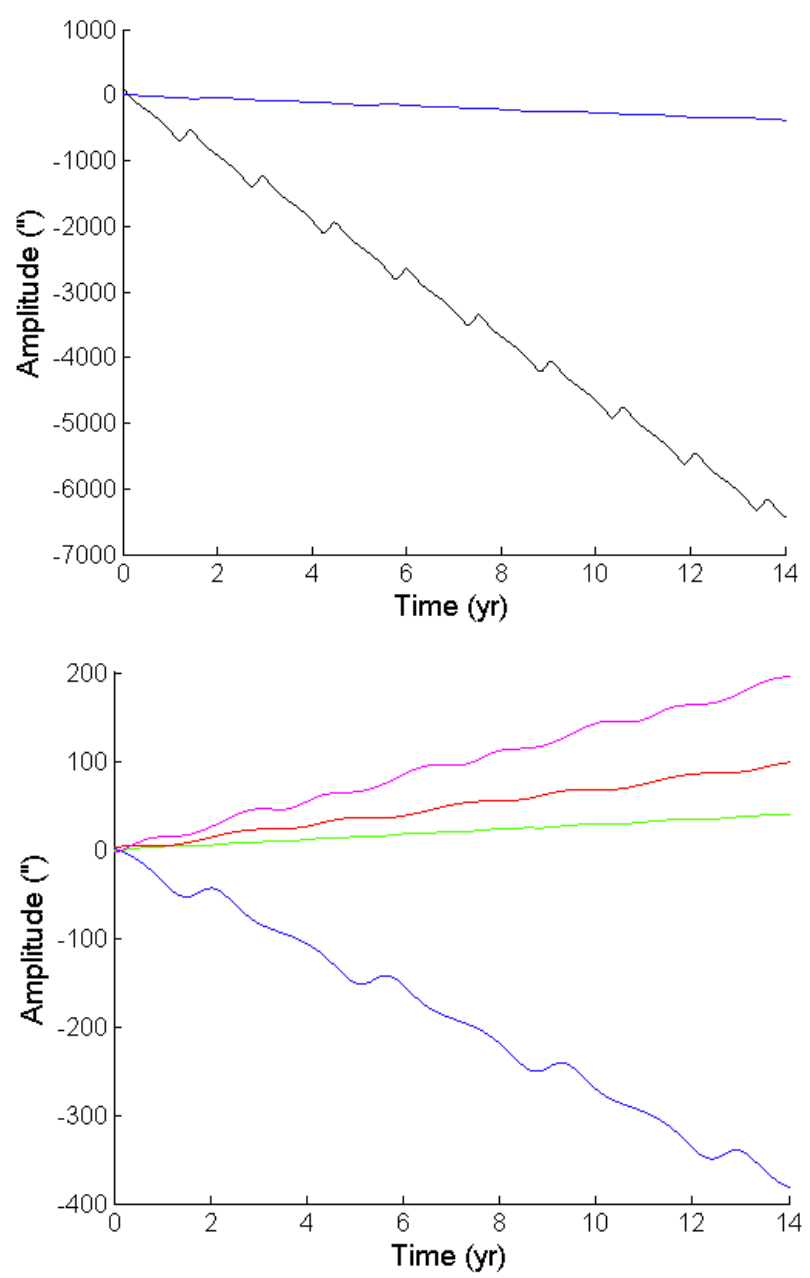

Fig. 4. Top: nutation motion in longitude added to the precession for (25143) Itokawa (black) and (2867) Steins (blue). Bottom: nutation motion in longitude added to the precession for (433) Eros (green), (2867) Steins (blue), (4) Vesta (pink), and (1) Ceres (red).

We also present the tables of nutation for (1) Ceres in Tables 7 and 8, which is the next target of the DAWN spacecraft. As already noted when commenting on Table 4, the amplitude of the nutation motion is fairly small. Nevertheless, it is close to the amplitude of the solar nutation on the Earth, that is to say, one arcsecond. 
Table 6. Nutation in obliquity for (4) Vesta.

\begin{tabular}{ccccc}
\hline \hline Sin $\left({ }^{\prime \prime}\right)$ & Cos $\left({ }^{\prime \prime}\right)$ & Amp $\left({ }^{\prime \prime}\right)$ & Period (year) & Arg \\
\hline-0.1263 & 0.1317 & 0.1823 & 3.6287 & $\mathrm{M}$ \\
1.3764 & -1.4321 & 1.9862 & 1.8143 & $2 \mathrm{M}$ \\
0.2898 & -0.3015 & 0.4182 & 1.2096 & $3 \mathrm{M}$ \\
0.0475 & -0.0494 & 0.0685 & 0.9072 & $4 \mathrm{M}$ \\
0.0072 & -0.0075 & 0.0104 & 0.7257 & $5 \mathrm{M}$ \\
0.0010 & -0.0011 & 0.0015 & 0.6048 & $6 \mathrm{M}$ \\
\hline
\end{tabular}

Table 7. Nutation in longitude for (1) Ceres.

\begin{tabular}{lcccc}
\hline \hline Sin $\left({ }^{\prime \prime}\right)$ & Cos $\left({ }^{\prime \prime}\right)$ & Amp $\left({ }^{\prime \prime}\right)$ & Period (year) & Arg \\
\hline 1.3362 & -0.1138 & 0.0915 & 4.6010 & $\mathrm{M}$ \\
-1.9198 & 1.4305 & 0.1634 & 2.3005 & $2 \mathrm{M}$ \\
-0.3591 & 0.2621 & 0.0303 & 1.5337 & $3 \mathrm{M}$ \\
-0.0515 & 0.0374 & 0.0043 & 1.1503 & $4 \mathrm{M}$ \\
-0.0068 & 0.0049 & 0.0006 & 0.9202 & $5 \mathrm{M}$ \\
-0.0068 & 0.0007 & 0.0001 & 0.7668 & $6 \mathrm{M}$ \\
\hline
\end{tabular}

Table 8. Nutation in obliquity for (1) Ceres.

\begin{tabular}{lcccc}
\hline \hline Sin $\left({ }^{\prime \prime}\right)$ & Cos $\left({ }^{\prime \prime}\right)$ & Amp $\left({ }^{\prime \prime}\right)$ & Period (year) & Arg \\
\hline 0.0078 & 0.0108 & 0.0133 & 4.6010 & $\mathrm{M}$ \\
-0.0979 & -0.1361 & 0.1676 & 2.3005 & $2 \mathrm{M}$ \\
-0.0179 & -0.0249 & 0.0307 & 1.5337 & $3 \mathrm{M}$ \\
-0.0026 & -0.0036 & 0.0044 & 1.1503 & $4 \mathrm{M}$ \\
-0.0003 & 0.0005 & 0.0006 & 0.9202 & $5 \mathrm{M}$ \\
-0.0000 & -0.0001 & 0.0001 & 0.7668 & $6 \mathrm{M}$ \\
\hline
\end{tabular}

Table 9. Amplitude of the nutation in longitude $\Delta \psi \sin \epsilon$ for (4) Vesta with our model (Cols. 1-2), and result given by Rambaux (2013) (Cols. 3-4).

\begin{tabular}{lccc}
\hline \hline \multicolumn{2}{c}{ Our model } & \multicolumn{2}{c}{ Rambaux (2013) } \\
Amp (mas) & Per (days) & Amp (mas) & Per (days) \\
\hline 1102.73 & 1325.3803 & 1091.97 & 1325.7251 \\
1715.42 & 662.6902 & 1718.27 & 662.8275 \\
366.95 & 441.7934 & 364.65 & 441.9033 \\
60.41 & 331.3451 & 59.59 & 331.4342 \\
9.24 & 265.0761 & 8.83 & 265.1506 \\
\hline
\end{tabular}

In the next step, we can compare our results with Rambaux (2013) who made calculations starting from an Eulerian formalism, instead of our Hamiltonian one. We developed the nutation at the fourth order of the eccentricity and as a sum of cosine and sine of arguments multiple of the mean anomaly of Vesta, whereas Rambaux (2013) give their results as a sum of cosine or sine of multiple of the longitude of (4) Vesta. The bias between the two kinds of arguments (mean anomaly and longitude) corresponds to the argument of the perihelion, which can be set as constant in our study as already pointed out.

Therefore although the arguments are different, we can compare the amplitude of motion by summing the squared values of cosine and sine of the previous table to evaluate the amplitude of the motion of nutation at a given frequency. We present the results obtained by our model and those by the semi-analytical model of Rambaux (2013) in Tables 7 and 8. However, it is appropriate to compare the terms over periods, and we find good agreement.

Finally for each asteroid, we compute the sole nutation part for both components $(\Delta \psi$ and $\Delta \epsilon)$ as a function of time. As a
Table 10. Amplitude of the nutation in longitude $\Delta \epsilon$ for (4) Vesta with our model (Cols. 1-2), and result given by Rambaux (2013) (Cols. 3-4).

\begin{tabular}{lccc}
\hline \hline \multicolumn{2}{c}{ Our model } & \multicolumn{2}{c}{ Rambaux (2013) } \\
Amp (mas) & Per (days) & Amp (mas) & Per (days) \\
\hline 182.36 & 1325.3803 & 181.51 & 1325.7251 \\
1986.24 & 662.6902 & 1987.23 & 662.8275 \\
418.24 & 441.7934 & 415.41 & 441.9033 \\
68.55 & 331.3451 & 67.59 & 331.4342 \\
10.41 & 265.0761 & 9.99 & 265.1506 \\
\hline
\end{tabular}

result we present in Fig. 5 the bidimensional nutation motion projected onto the equatorial plane for each asteroid. In all cases the spin axis describes a periodic closed loop whose amplitude is altered mainly by the value of the obliquity. The structure of the loop is obviously generated by the combination of two or three leading sinusoidal components. The scales along the axes have been adjusted according to the amplitudes. We point out that for (433) Eros and (25143) Itokawa, the amplitudes are significantly large at the level of $30^{\prime \prime}$ and $60^{\prime \prime}$, respectively, whereas they are on the order of $1^{\prime \prime}$ or less for the three other bodies. In the case of (433) Eros, our results in particular the feature of the nutation loop, agree completely with the previous study by Souchay et al. (2003a). This confirms the validity of our computations.

\section{Conclusion}

In this paper, we have applied an analytical model to calculating the precession-nutation components of the rotational motion of five asteroids based on Kinoshita's theory (Kinoshita 1977). Four of the asteroids, (4) Vesta, (433) Eros, (2867) Steins, and (25143) have been explored by a space probe and in consequence offer very accurate physical and rotational parameters as used in our computations, such as the moments of inertia, the orientation of the pole axis and the spin angular rate. (1) Ceres will soon be subjected to a flyby by the DAWN probe, but its parameters above are already very well constrained by ground-based observations. In consequence, as an output of our computations, we could show the evolution of the obliquity and precession of the longitude of the node of each asteroid very accurately, as well as the loops described by their polar axis by taking the sole effect of the nutation. These results can be considered as a continuation of the work done by Lhotka et al. (2013) for a larger set of 100 asteroids but based on less accurate data for the majority of these 100 asteroids.

It is true that the effects underlined here will maybe stay below the detection level, as already been pointed out by Rambaux et al. (2011, 2013) for (1) Ceres and (4) Vesta. Nevertheless, we show that for instance in the case of (25143) Itokawa, the general precession rate $\dot{\psi}=-(461.52 \pm 6.57)^{\prime \prime} / \mathrm{yr}$ corresponds to roughly $1 \%$ in eight years and might be detectable within a rather small time interval. Our analytical expresions used here could be applied to any other asteroid in the future, once we know its physical and rotational characteristics. We restricted our developments here to the fourth order of the eccentricity, but they could be easily extended to the highest order.

At last some extensions of this work could be carried out, for instance, by evaluating the Oppolzer terms that separate the motion of the axis of figure from the one of axis of rotation, or by studying the effect of the triaxial comonent of the potential, done by Souchay et al. (2003b) for (433) Eros. Long-term study of the rotational evolution should also be undertaken by numerical integration following the same canonical equations as in this paper 

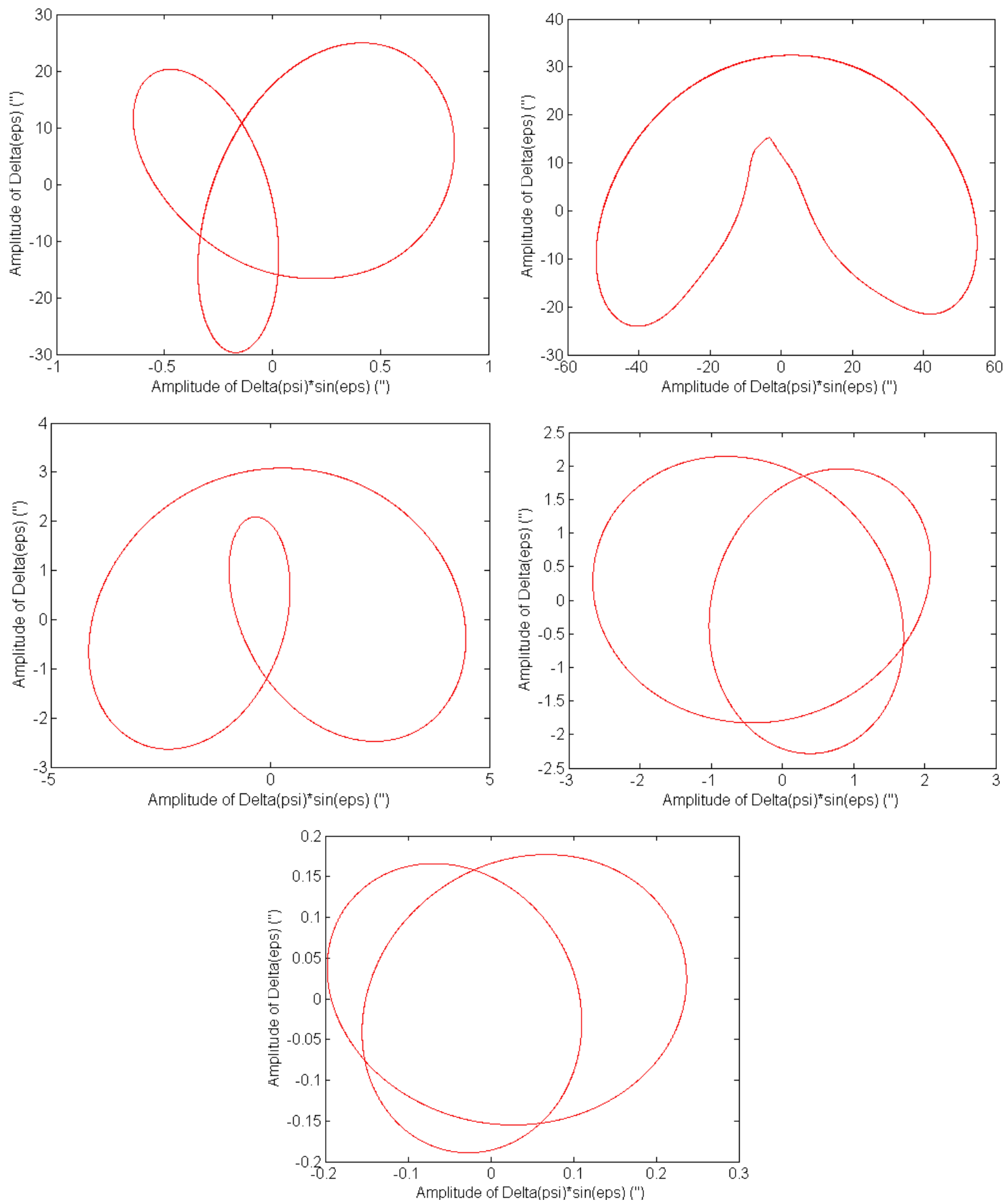

Fig. 5. Bi-dimensional nutation motion projected onto the equatorial plane. From top to bottom and from left to right: (433) Eros, (25143) Itokawa, (2867) Steins, (4) Vesta and (1) Ceres.

but by taking the direct perturbing effect of the planets into account, as well as their indirect effect on the orbit of the asteroid itself.

\section{References}

Asmar, S. W., Konopliv, A. S., Park, R. S., et al. 2012, European Planetary Science Congress, 814

Bills, B. G., \& Nimmo, F. 2011, Icarus, 213, 496

Bouquillon, S., \& Souchay, J. 1999, A\&A, 345, 282

Carry, B., Dumas, C., Fulchignoni, M., et al. 2008, A\&A, 478, 235

Castillo-Rogez, J. C., \& McCord, T. B. 2010, Icarus, 205, 443

Chamberlain, M. A., Sykes, M. V., \& Esquerdo, G. A. 2007, Icarus, 188, 451

Cottereau, L., Souchay, J., \& Aljbaae, S. 2010, A\&A, 515, A9

Demura, H., Kobayashi, S., Nemoto, E., et al. 2006, Science, 312, 1347

Hori, G. 1966, PASJ, 18, 287
Hughes, S. 1981, Celest. Mech., 25, 101

Jorda, L., Lamy, P. L., Gaskell, R. W., et al. 2012, Icarus, 221, 1089

Kinoshita, H. 1977, Celest. Mech., 15, 277

Konopliv, A. S., Asmar, S. W., Bills, B. G., et al. 2011, Space Sci. Rev., 163, 461

Lara, M., Fukushima, T., \& Ferrer, S. 2011, MNRAS, 415, 461

Lhotka, C., Souchay, J., \& Shahsavari, A. 2013, A\&A, 556, A8

McCord, T. B., Castillo-Rogez, J., \& Rivkin, A. 2011, Space Sci. Rev., 163, 63

Miller, J. K., Konopliv, A. S., Antreasian, P. G., et al. 2002, Icarus, 155, 3

Rambaux, N. 2013, A\&A, 556, A151

Rambaux, N., Castillo-Rogez, J., Dehant, V., \& Kuchynka, P. 2011, A\&A, 535, A43

Souchay, J., \& Bouquillon, S. 2005, A\&A, 433, 375

Souchay, J., Loysel, B., Kinoshita, H., \& Folgueira, M. 1999, A\&AS, 135, 111

Souchay, J., Folgueira, M., \& Bouquillon, S. 2003a, Earth Moon Planets, 93, 107 Souchay, J., Kinoshita, H., Nakai, H., \& Roux, S. 2003b, Icarus, 166, 285 